\title{
Improved Two-Level Model Averaging Techniques in Drosophila Brain Modeling
}

\author{
Cheng-Chi Wu ${ }^{1}$, Chao-Yu Chen ${ }^{1}$, Hsiu-Ming Chang ${ }^{2}$, Ann-Shyn Chiang ${ }^{2}$, \\ and Yung-Chang Chen ${ }^{1}$ \\ ${ }^{1}$ Department of Electrical Engineering \\ ${ }^{2}$ Department of Life Science \\ National Tsing Hua University, \\ 101, Section 2, Kuang-Fu Road, Hsinchu, Taiwan, R.O.C. \\ \{dennis, ordnance, ycchen\} @benz . ee.nthu . edu.tw, \\ \{hmchang, aschiang\} alife.nthu.edu.tw
}

\begin{abstract}
Two-level model averaging techniques have been proposed to construct the 3D reference template for the Drosophila brain. The surface-based reference template is suitable for integration of experimental data from different laboratories. The 3D distance transform is the most memory and time consuming part in the model averaging algorithm. With the improvement of microscopic scanning technology, images of higher resolution can be acquired. Thus, the memories required for 3D distance transform become critical. In this paper, improved two-level model averaging techniques are proposed with three improvements. A two-scale distance map creation algorithm is introduced to reduce the memory cost in the distance transform. The computational time is reduced by a reduction of computation points in the distance map creation. The third improvement is an outlier rejection module to improve the robustness of the resulting average model.
\end{abstract}

Keywords: Model averaging, Drosophila, surface-based.

\section{Introduction}

In the field of neuroscience, atlas of the specific structure is pursued in many applications. Especially in the brain research, brain atlases are needed for studies of laboratory animals and human brain. The brain atlas could be either a representative individual brain [1] or an average model generated from a number of datasets [2]. Since the individual variability exists in all species, the average model is preferred.

Methods for averaging multiple image datasets can be categorized into two groups: the voxel-based averaging algorithms and the surface-based averaging algorithms. The voxel-based averaging algorithms are typically based on local decision fusion schemes. Voxel-based probabilistic atlases [3,4] have been proposed for the honeybee brain and the Drosophila brain. A probability atlas provides only a boundary for statistical confidence instead of an absolute anatomic shape and position. It is suitable for distinguishing normal brains from disease specific ones. 
For serving as a reference template for data integration, a surface-based atlas is preferred. In the Drosophila brain atlas, data from different laboratories can be integrated via a surface warping with specific landmarks, such as the brain cortex or specific neuropils. Two-level model averaging techniques [5] are introduced to construct the average shape model for the cortex and neuropils of the Drosophila brain. The first step is coarse-level model averaging. Global characteristics of different individual models are averaged in this step. After coarse-level model averaging, each individual model is transformed into a corresponding pseudo-average model.

In the fine-level model averaging step, the average model can be obtained by determining the shape average of the globally-registered pseudo-average models. A 3D distance field [6] is utilized to generate the signed distance map for each pseudoaverage model.

Many efforts have been made on building a signed distance map of a triangular mesh. The procedure can be separated into two parts: sign determination and distance transformation. In the first half, a famous method is to count the intersection of a ray going from the given point to infinity. The point is determined to be within the model if the intersection number is odd and vice versa [7]. Another well known method utilizes the normal of the closest feature on mesh of the given point. Angle weighted pseudo normal method [8] computes the normal of all faces, edges, and points on the triangular mesh. In the distance transformation part, brute force algorithm directly computes all of the distance from the given point to the mesh points. Many methods have been proposed to improve the computational efficiency [9].

In the two-level model averaging algorithm, a linear time algorithm for distance transformation is applied. With the improvement of microscopic scanning technology, images of higher resolution can be acquired. The memories required for computing the distance transformation become the bottleneck of the system when the source images are of high resolution. In the mean while, the increase of time consumption for the algorithm is in proportion to the number of points needed for distance transformation.

In this paper, improved two-level model averaging techniques are proposed to generate the average model from a set of triangular mesh models. Three improvements are made in the proposed algorithm. First, a two-scale distance map creation is introduced to reduce the memory cost. Second, the computational time consumption is reduced by a point-reduction scheme in the distance transformation. Third, an outlier rejection procedure is implemented to improve the robustness of the proposed algorithm.

The paper is organized as follows. Section 2 overviews the proposed algorithms. Section 3 and 4 describe the coarse-level and the fine-level model averaging accordingly. The outlier rejection procedure is presented in Section 5. Section 6 contains experimental results and conclusions are made in Section 7.

\section{System Overview}

Original datasets used in this study are confocal microscopic image slices. An experienced expert helps to label the object to be averaged from the image slices. The object boundary in each image is assumed to be composed of several non-crossing simple closed contours. 3D surface models of the objects are constructed from these 
labeled sequential parallel 2D sections by the algorithm describes in [10]. The proposed algorithm is trying to build an average 3D surface model from a group of individual datasets.

The system flowchart of the two-level model averaging algorithm is shown in Fig. 1. The goal of the coarse-level model averaging is to register the global characteristic of different individual models, such as orientations and positions. The candidate of the reference model is chosen to be the individual model which has the volume size closest to the average volume size. Each individual model is transformed into a corresponding pseudo-average model after coarse-level model averaging.

The next step is the fine-level model averaging. A signed distance map is built for each pseudo-average model. After cumulating the signed distance maps of all pseudoaverage models, the surface of a pro forma average model can be extracted by zerocrossing detection of the cumulative distance field.

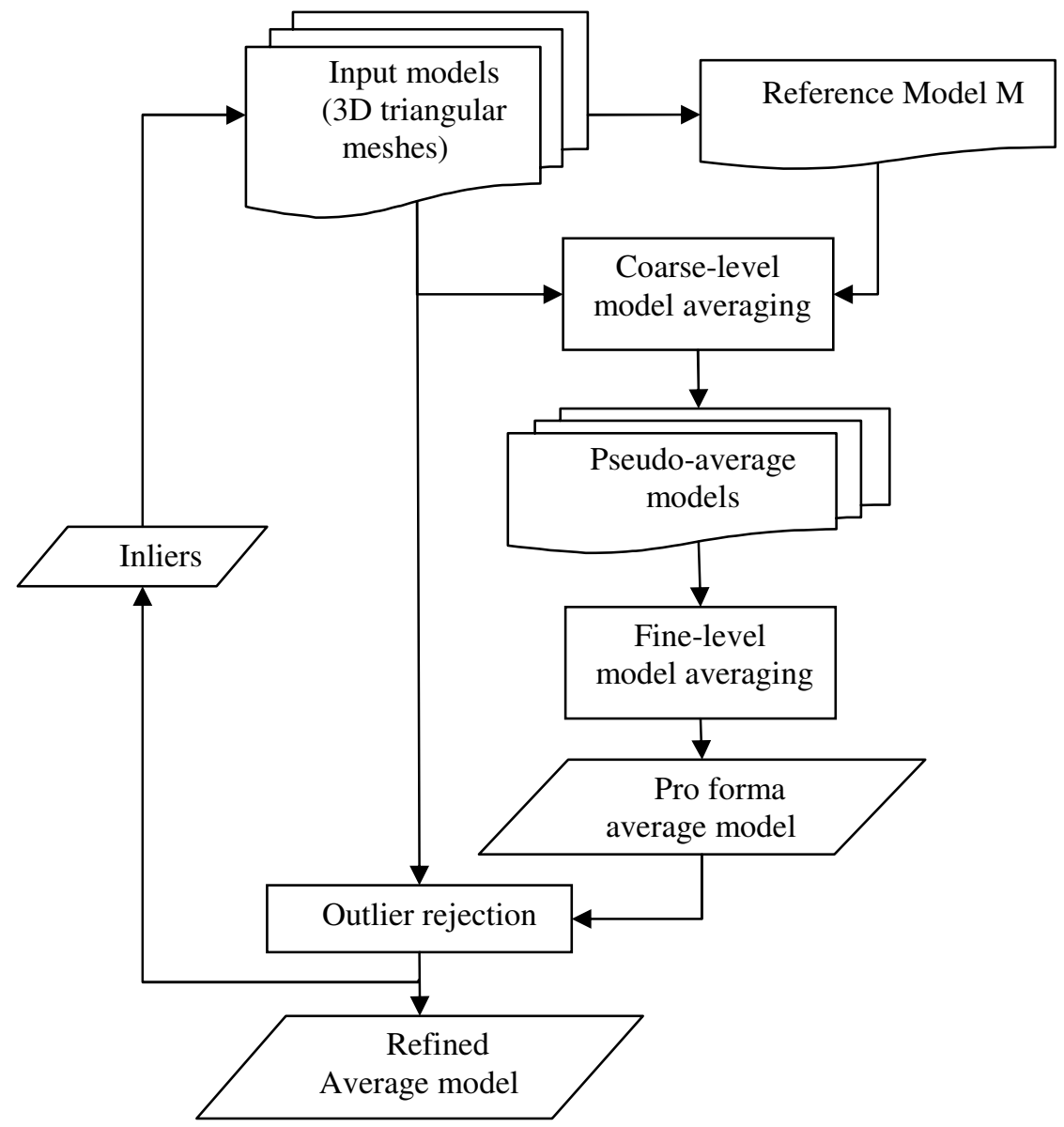

Fig. 1. System flowchart of the improved two-level model averaging algorithm 
The final step is an outlier rejection to improve the robustness of the average model. Outliers can be picked out by an outlier rejection procedure. A refined average model can be obtained by performing the improved two-level model averaging algorithm with the remaining inliers.

\section{Coarse-Level Model Averaging}

The coarse-level model averaging is implemented by a rigid registration. In general, the affine registration can achieve better alignments than that of the rigid registration. However, the shearing and scaling are two unwanted properties in the coarse-level model averaging. The affine registration is easily biased by an improper reference model. If the reference model is a tilted model, all other individual models will be tilted after transformation.

Instead, the rigid registration allows only translation and rotation of the target model. The geometry of the model is preserved under rigid transformation. For globally registering the individual models, the rigid registration is a reliable choice.

A rigid transformation is define as:

$$
\mathbf{x}^{\prime}=\mathbf{R}(\mathbf{x}-\mathbf{C})+\mathbf{C}+\mathbf{T} .
$$

where $\mathbf{x}$ is the position vector of the original point on the target model. $\mathbf{x}^{\prime}$ is the position vector of the transformed point. $\mathbf{C}=\left[C_{x}, C_{y}, C_{z}\right]^{T}$ is the center of rotation. $\mathbf{T}=\left[T_{x}, T_{y}, T_{z}\right]^{T}$ is the translation matrix. $\mathbf{R}$ is the rotation matrix, which is represented by the product of three elementary rotation matrices

$$
\mathbf{R}=\mathbf{R}_{x} \mathbf{R}_{y} \mathbf{R}_{z} .
$$

where $\mathbf{R}_{x}, \mathbf{R}_{y}$, and $\mathbf{R}_{z}$ are defined by

$$
\mathbf{R}_{x}=\left[\begin{array}{ccc}
1 & 0 & 0 \\
0 & \cos \theta_{x} & \sin \theta_{x} \\
0 & -\sin \theta_{x} & \cos \theta_{x}
\end{array}\right], \mathbf{R}_{y}=\left[\begin{array}{ccc}
\cos \theta_{y} & 0 & \sin \theta_{y} \\
0 & 1 & 0 \\
-\sin \theta_{y} & 0 & \cos \theta_{y}
\end{array}\right], \mathbf{R}_{z}=\left[\begin{array}{ccc}
\cos \theta_{z} & \sin \theta_{z} & 0 \\
-\sin \theta_{z} & \cos \theta_{z} & 0 \\
0 & 0 & 1
\end{array}\right] .
$$

The average distance between target and reference model is defined as:

$$
D=\frac{1}{N} \sum_{i=1}^{N} d^{2}\left(\mathbf{x}^{\prime}\right)
$$

where $d^{2}\left(\mathbf{x}^{\prime}\right)$ is the distance between $\mathbf{x}^{\prime}$ and the reference model and $N$ is the number of points on the target model.

Let $\mathbf{p}=\left(T_{x}, T_{y}, T_{z}, \theta_{x}, \theta_{y}, \theta_{z}\right)$ denotes the parameters of the rigid transformation. Since $D$ is a function of $\mathbf{p}$, the parameters of the rigid transformation can be solved by minimizing the average distance $D(\mathbf{p})$ via the following iteration: 


$$
\mathbf{p}^{\prime}=\mathbf{p}-\delta \cdot \nabla D(\mathbf{p}) .
$$

where $\delta$ is the step size of iteration and

$$
\nabla D(\mathbf{p})=\left[\frac{\partial D(\mathbf{p})}{\partial \theta_{x}} \frac{\partial D(\mathbf{p})}{\partial \theta_{y}}, \frac{\partial D(\mathbf{p})}{\partial \theta_{z}}, \frac{\partial D(\mathbf{p})}{\partial T_{x}} \frac{\partial D(\mathbf{p})}{\partial T_{y}}, \frac{\partial D(\mathbf{p})}{\partial T_{z}}\right]
$$

An individual model is transformed into a corresponding pseudo-average model by applying a rigid transformation with the parameters solved in (5).

\section{Fine-Level Model Averaging}

The final average model can be obtained by fine-level model averaging of the pseudoaverage models. The shape-based averaging method [11] is implemented on the triangular meshes. A signed distance map $\operatorname{SDM}(\vec{x})_{i}$ is built for each pseudo-average model $M_{i} . \operatorname{SDM}(\vec{x})_{i}$ is defined as:

$$
\operatorname{SDM}(\vec{x})_{i}= \begin{cases}+\min _{\vec{x}_{i} \in \text { surface }} d\left(\vec{x}, \vec{x}_{i}\right), & \text { if } \overrightarrow{\mathrm{x}} \text { is outside the model } \\ -\min _{\vec{x}_{i} \in \text { surface }} d\left(\vec{x}, \vec{x}_{i}\right), & \text { if } \overrightarrow{\mathrm{x}} \text { is inside the model }\end{cases}
$$

where $d\left(\vec{x}, \vec{x}_{i}\right)$ is the Euclidean distance between $\vec{x}$ and $\vec{x}_{i}$. Therefore, $\operatorname{SDM}(\vec{x})_{i}$ contains the information that either $\vec{x}$ is inside or outside the model $M_{i}$, and how far it is from the surface boundary. $\vec{x}$ is on the surface if $\operatorname{SDM}(\vec{x})_{i}=0$.

The cumulative signed distance map $R(\vec{x})$ is defined as:

$$
R(\vec{x})=\sum_{i=1}^{K} \operatorname{SDM}(\vec{x})_{i}
$$

where $K$ is the number of pseudo-average models. The surface of the final average model can be extracted by zero-crossing detection of $R(\vec{x})$.

A 2D example is shown is Fig. 2. The inputs are two ellipses (Fig. 2(a) and 2(b)). The signed distance maps is computed for both ellipses and drawn in $3 \mathrm{D}$ (Fig. 2(c) and 2(d)). The cumulative signed distance map is shown in Fig. 2(e). The averaging result of the inputs is a flattened ellipse (Fig. 2(f)).

In [6], a linear time algorithm is proposed for computing the Euclidean distance transform. The algorithm has a serial computational complexity linear in the number of image pixels. However, memories required for storing the signed distance map depend on the voxel number of the model. The memory cost becomes the bottleneck of the system when the source images are of high resolution. A two-scale distance map creation algorithm is proposed to reduce the memory cost. 
A point-reduction scheme is introduced to reduce the time expense for computing the distance transform. This is achieved by computing the distance transform only on the necessary voxels.

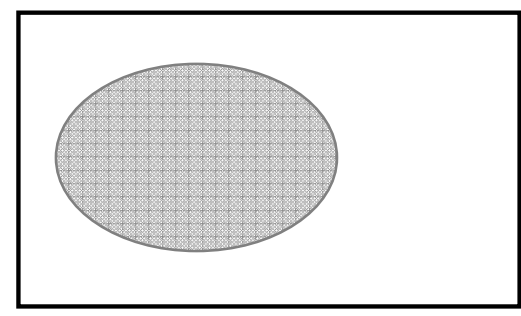

(a)

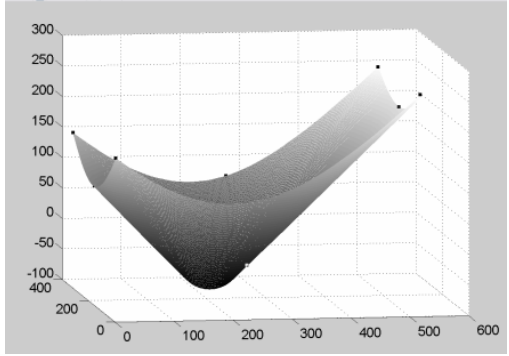

(c)

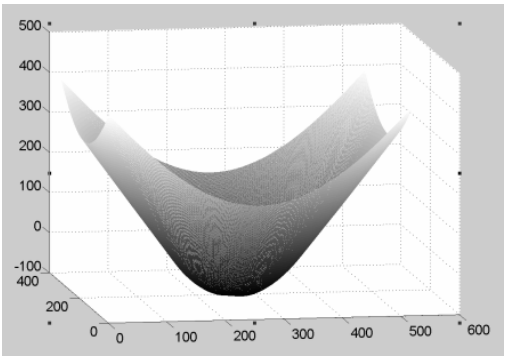

(e)

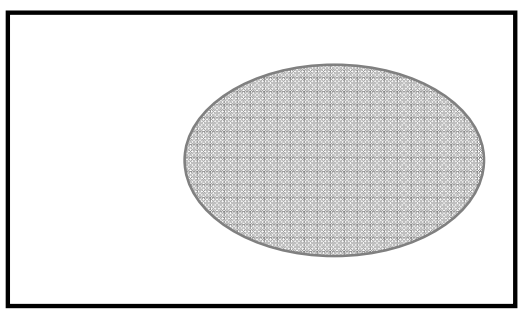

(b)

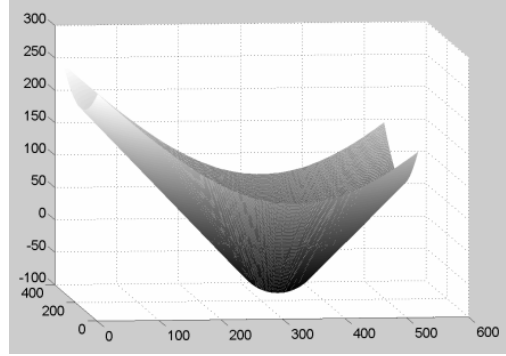

(d)

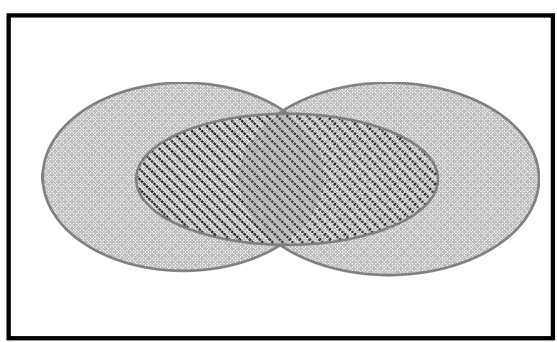

(f)

Fig. 2. A $2 \mathrm{D}$ example of model averaging. (a) and (b): two input ellipses. (c) and (d): the corresponding signed distance map of (a) and (b). (e): the cumulative signed distance map. (f): the averaging result (ellipse with oblique lines) of two ellipses.

\subsection{Two-Scale Distance Map Creation}

A two-scale distance map creation algorithm is proposed to reduce the memory cost by a factor of $\beta^{3}$. The idea is to compute the distance transform in two steps. Denote $O(v)$ as the voxel space of the object $O$. In the first step, $O(v)$ is down-sampled by a factor of $\beta$ in each axis. The down-sampled voxel space of the object is denoted as 
$O_{\beta}(v)$. The distance map of $O_{\beta}(v)$ can be generated as $D M_{\beta}$. The exact distance transform of the voxel on the original scale can be computed only in a small region.

A 2D example is shown in Fig. 3. The object $O$ is the ellipse. The hollow circles around the ellipse are the down-sampled object $O_{\beta}(v)$. The distance map of $O_{\beta}(v)$ is calculated on the grid points. Assuming the exact distance transform of a certain voxel, point $G$, on the original scale is to be computed. The rough distance map of the down-sampled space is generated.

In the down-sampled space, the neighborhood of $G$ is the point set $\{\mathrm{A}, B, C$ and $D\}$. Closest feature point set on the ellipse is $\{\mathrm{E}, F\}$. The exact distance transform on the original scale can be found in a small region, which is the up-sampled space encloses $A C E F D B$.

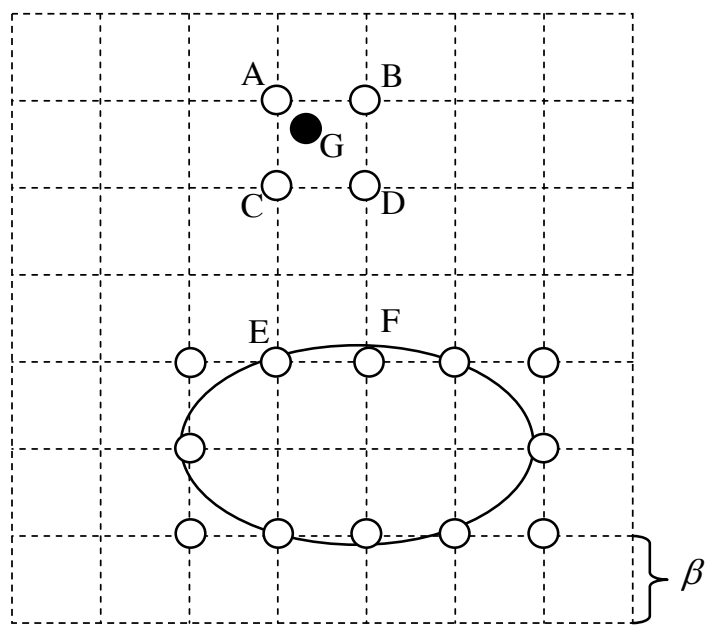

Fig. 3. An ellipse down-sampled by a factor of $\beta$. E and $F$ are the closest feature points of $\{\mathrm{A}, B, C$ and $D\}$.

\subsection{Point-Reduction Scheme in the Distance Transform}

The computational time consumption is reduced by a point-reduction scheme in the distance transformation. Recall that the surface of the final average model is extracted by zero-crossing detection of the cumulative signed distance map. After coarse-level model averaging, the pseudo-average models are aligned to the same coordinate space. Voxels in this coordinate spaces can be classified into three groups: 1) voxels inside every models; 2 ) voxels outside every models; 3 ) the remaining voxels.

For the first group of voxels, the value of the signed distance map of the same voxel is negative for all pseudo-average models. For the second group of voxels, the value of the signed distance map of the same voxel is positive for all pseudo-average models. Thus, the cumulative distance map of such voxels will never be zero. In other 
words, the first and second group of voxels well not be on the surface boundary of the average model. These voxels can be assigned to be inside or outside of the average model directly.

Only the third group of voxels need to compute distance transform. The twoscale distance map creation method is applied on these voxels for each pseudo-average model.

\section{Outlier Rejection}

The rejection of outliers is based on the distance information. Let $I_{i}$ be the $i$ th individual model and $K$ be the number of individual models. The average model, $M_{\text {avg }}$, can be generated by the proposed improved two-level model averaging techniques. A corresponding distance map for $M_{\text {avg }}$ can be built subsequently. To determine whether model $I_{i}$ is an outlier, $I_{i}$ is first rigidly registered to $M_{a v g}$. For every point on $I_{i}$, the minimum distance from it to $M_{\text {avg }}$ can be computed. The mean distance between $I_{i}$ and $M_{\text {avg }}$ can be calculated by the following equation:

$$
\bar{x}_{i}=\frac{1}{N} \sum_{i=1}^{N} D\left(x_{i}\right)
$$

where $x_{i}$ is the point on $I_{i}$ and $N$ is the number of points in $I_{i} \cdot \bar{x}_{i}$ is the mean distance between $I_{i}$ and $M_{\text {avg }}$.

The mean distance of all individual models can be computed serially. The mean distance of all individual models can be defined as:

$$
\bar{X}=\frac{1}{K} \sum_{i=1}^{K} \bar{x}_{i} .
$$

The standard deviation of all individual models can be computed by the following equation:

$$
\sigma=\sqrt{\frac{1}{K} \sum_{i=1}^{K}\left(\bar{x}_{i}-\bar{X}\right)^{2}} .
$$

Finally, the model is regarded as an outlier if the following inequality is satisfied:

$$
\begin{cases}\bar{x}_{i}>\bar{X}+\sigma, & \text { if } \sigma \geq T_{H} . \\ \bar{x}_{i}>\bar{X}+T_{H}, & \text { if } \sigma<T_{H} .\end{cases}
$$

where $T_{H}$ is a pre-defined threshold. 


\section{Experimental Results}

There are 34 datasets of female Drosophila used in this study. Fig. 4 shows the results for model averaging of the brain cortex. The superposition of 34 pseudo-average models after coarse-level averaging is shown in Fig. 4(a). The average model and the superposition of 34 pseudo-average models are depicted in Fig. 4(b).

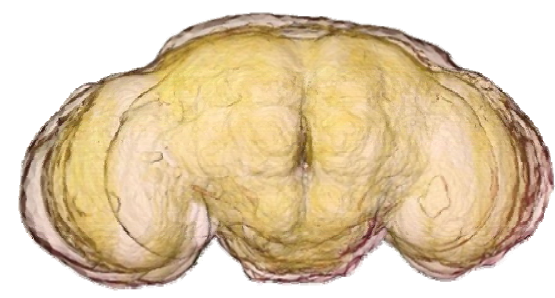

(a)

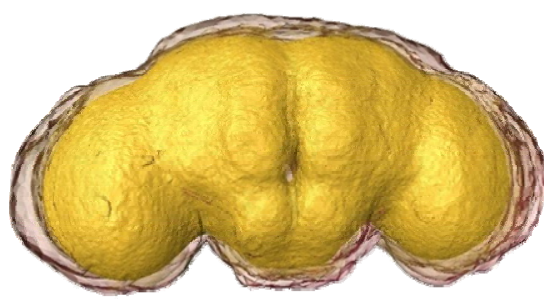

(b)

Fig. 4. The results for model averaging of the brain cortex. (a): 34 pseudo-average models after coarse-level model averaging. (b): The average model (opaque) and the superposition of 34 pseudo-average models (transparent).

Fig. 5 illustrates the point-reduction scheme and the two-scale hierarchy in the distance transform. Given pseudo-average models, Fig. 5(a) shows the points needed to compute the distance transform in a slice. The corresponding region for the twoscale distance map creation is depicted in Fig. 5(b).

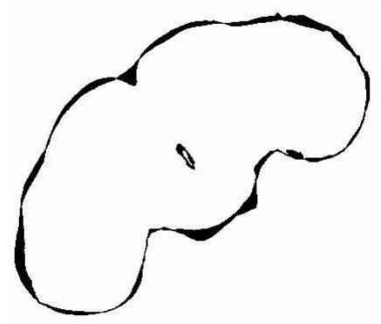

(a)

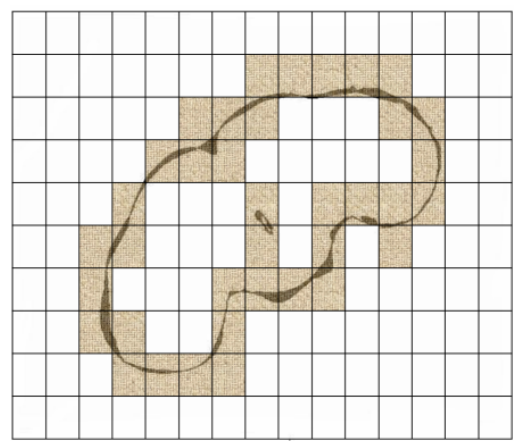

(b)

Fig. 5. Example of the point-reduction scheme and the two-scale hierarchy in the distance transform. (a) The points needed to compute the distance transform. (b) The corresponding region for the two-scale distance map creation.

The performance improvement in the distance transform is shown in Table 1. Measurements were obtained using a workstation with Intel dual Xeon $2.0 \mathrm{GHz} \mathrm{CPU}$. This workstation was equipped with 4 GBytes of memory. The linear time distance transform algorithm will be out of memory for images constructed from the high 
Table 1. The performance improvement of distance transform

\begin{tabular}{ccc}
\hline Voxel number & $\begin{array}{c}\text { The linear time distance } \\
\text { transform algorithm }\end{array}$ & $\begin{array}{c}\text { Two-scale and point-reduction } \\
\text { in distance transform }\end{array}$ \\
\hline $320 \times 327 \times 139$ & 13343 (milliseconds) & 10032 (milliseconds) \\
$600 \times 614 \times 206$ & Out of memory & 34735 (milliseconds) \\
$900 \times 880 \times 289$ & Out of memory & 43157 (milliseconds) \\
\hline
\end{tabular}

resolution confocal microscopy. With the improvements in distance transform, both memory and time consumption can be reduced.

The results of outlier rejection is shown in Fig. 6. Four outliers are detected by the outlier rejection algorithm.

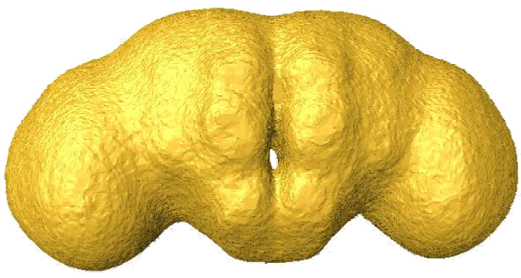

(a)
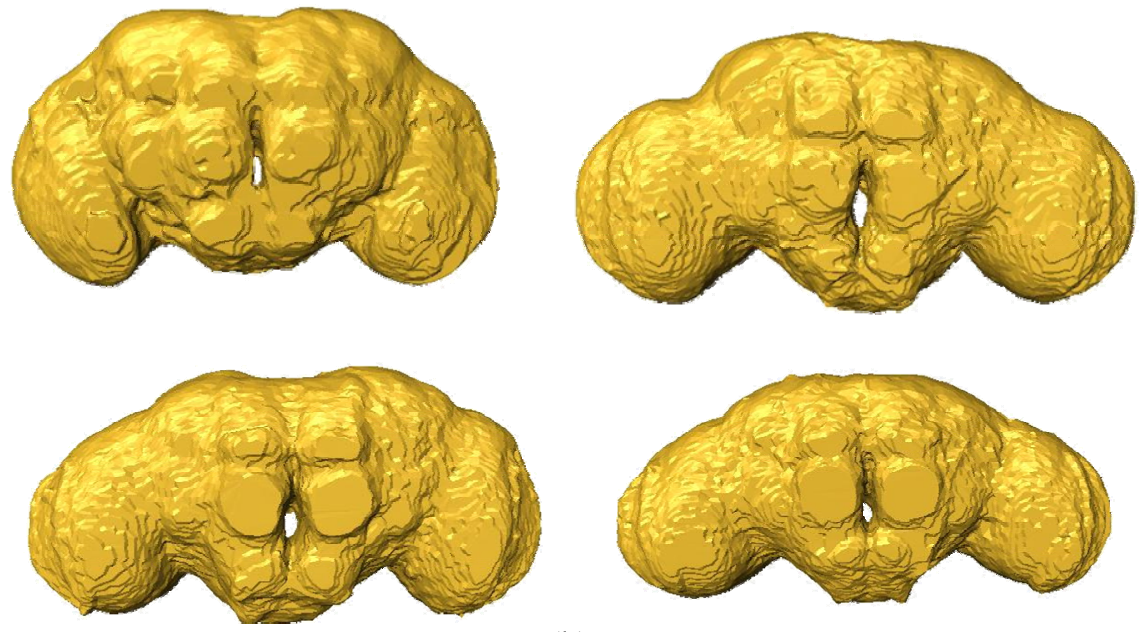

(b)

Fig. 6. (a) The average model of the 34 datasets. (b) The outliers: no. 12 , no. 29 , no. 30, and no. 31 .

\section{Conclusions}

In this paper, an improved two-level model averaging algorithm is proposed for the Drosophila brain modeling. Two improvements are made in the fine-level model averaging. A two-scale distance map creation is introduced to reduce the memory cost. The computational time consumption is reduced by a point-reduction scheme in 
the distance transform. With these two improvements, average model can be constructed from high resolution confocal microscopic images.

An outlier rejection procedure is implemented to improve the robustness of the model averaging algorithm. A refined average model can be obtained by performing the improved two-level model averaging algorithm with the remaining inliers.

\section{References}

1. Talairach, J., Tournoux, P.: Coplanar Stereotaxic Atlas of the Human Brain. Thieme Medical, New York (1988)

2. Friston, K.J., Holmes, A.P., Worsley, K.J., Poline, J.P., Frith, C.D., Frackowiak, R.S.J.: Statistical Parametric Maps in Functional Imaging: A general Linear Approach. Human Brain Mapping 2, 189-210 (1995)

3. Brandt, R., Rohlfing, T., Rybak, J., Krofczik, S., Maye, A., Westerhoff, M., Hege, H.-C., Menzel, R.: Three-Dimensional Average-Shape Atlas of the Honeybee Brain and its Applications. J. Comp. Neurol. 492, 1-19 (2005)

4. Rein, K., Zockler, M., Mader, M.T., Grubel, C., Heisenberg, M.: The Drosophila Standard Brain. Current Biology 12, 227-231 (2002)

5. Chen, Y.C., Chen, Y.C., Chiang, A.S.: Two-Level Model Averaging Techniques in Dorsophila Brain Imaging. In: Proceedings of 2002 IEEE International Conference on Image Processing, vol. 2, pp. 941-944. Rochester, New York (2002)

6. Maurer, C.R., Qi, R., Raghavan, V.: A linear time algorithm for computing exact Euclidean distance transforms of binary images in arbitrary dimensions. IEEE Trans. on Pattern Analysis and Machine Intelligence 25, 265-270 (2003)

7. Dachille, F., Kaufman, A.: Incremental Triangle Voxelization. In: Proc. Graphics Interface, pp. 205-212 (2000)

8. Baerentzen, J.A., Aanaes, H.: Signed Distance Computation Using the Angle Weighted Pseudonormal. IEEE Trans. on Visualization and Computer Graphics 11(3), 243-253 (2005)

9. Gue'ziec, A.: Meshsweeper: Dynamic Point-to-Polygonal Mesh Distance and Applications. IEEE Trans. on Visualization and Computer Graphics 7(1), 47-60 (2001)

10. Chen, Y.C., Chen, Y.C., Chiang, A.S., Hsieh, K.S.: A Reliable Surface Reconstruction System in Biomedicine. Computer Methods and Programs in Biomedicine 86(2), 141-152 (2007)

11. Rohlfing, T., Maurer, C.R.: Shape-Based Averaging. IEEE Trans. on Image Proc. 16(1), 153-161 (2007) 